Cipango Cahiers d'études japonaises

$15 \mid 2008$

Guerre, colonialisme et commémoration

\title{
Autour de quelques os
}

La mémorialisation des criminels de guerre de catégorie A

Around some Bones: the Memorialization of A-class War Criminals

\section{Michael Lucken}

\section{(2) OpenEdition}

Journals

\section{Édition électronique}

URL : https://journals.openedition.org/cipango/215

DOI : $10.4000 /$ cipango. 215

ISSN : 2260-7706

\section{Éditeur}

INALCO

\section{Édition imprimée}

Date de publication : 1 janvier 2008

Pagination : 101-122

ISBN : 978-2-85831-177-4

ISSN : 1164-5857

Référence électronique

Michael Lucken, « Autour de quelques os », Cipango [En ligne], 15 | 2008, mis en ligne le 11 novembre 2011, consulté le 30 juin 2021. URL : http://journals.openedition.org/cipango/215 ; DOI : https:// doi.org/10.4000/cipango.215

Ce document a été généré automatiquement le 30 juin 2021.

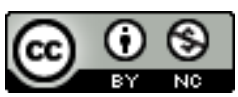

Cipango est mis à disposition selon les termes de la Licence Creative Commons Attribution - Pas d'Utilisation Commerciale 4.0 International. 


\title{
Autour de quelques os
}

\author{
La mémorialisation des criminels de guerre de catégorie A \\ Around some Bones: the Memorialization of A-class War Criminals
}

\author{
Michael Lucken
}

1 Lorsque furent exécutés sept « criminels de guerre de catégorie $\mathrm{A}$ » $(\bar{E}-k y \bar{u}$ senpan $\mathrm{A}$ 級 戦犯) dans la nuit du 23 décembre 1948, les autorités américaines avaient en tête de faire disparaître leurs ossements comme les Soviétiques et les Alliés l'avaient fait en Allemagne pour Hitler, Goebbels, Himmler ou Göring. Ne rien conserver des corps était dans l'esprit des vainqueurs le meilleur moyen d'éviter que des reliques et donc un lieu précis cristallisent le souvenir de ces hommes et de leur idéologie. Même si les nombreux groupes et sympathisants néo-nazis entretiennent un actif marché des militaria et des souvenirs du troisième Reich, il faut reconnaître que le procédé a fonctionné de façon relativement efficace. Il y a certes en Allemagne une multitude de lieux qui peuvent servir de supports à la mémorialisation (Berstesgaden, la brasserie Hofbraühaus à Munich, le forum du Reichsparteigelände à Nuremberg), mais aucun n'ayant une légitimité supérieure aux autres, ils sont en situation de concurrence et ne parviennent pas à porter véritablement le souvenir. Néanmoins, au Japon, les restes des sept principaux criminels de guerre exécutés après le Procès de Tōkyō ont finalement été partiellement conservés. L'objectif du présent article est de montrer comment à partir de la présence de quelques restes infimes mais bien concrets s'est constitué progressivement un ensemble mémoriel relativement étendu qui perpétue l'action de ces hommes et l'idéologie qui leur est rattachée.

2 Les processus mémoriaux ne sont pas des processus abstraits. Ils fonctionnent comme des entités vivantes. Ils dépendent, certes, d'un environnement propice à leur développement, mais ce sont aussi des entités corporelles. Leur étude implique donc à la fois la prise en compte d'un ekos et d'un bios, d'un milieu transformant que l'histoire sert à décrire, et d'une matérialité active que l'esthétique découvre. L'objet mémoriel possède en effet toujours une forme, une substance. Pourtant, celle-ci est très souvent mal comprise et mal analysée par les historiens. Comme s'il y avait d'un côté l'idée mémorielle, le pur souvenir de l'événement et, de l'autre, des objets qui ne seraient que des lieux ou des supports transitoires. Or, s'il y a bien des réseaux qui donnent 
l'impression que le souvenir est dématérialisé, ces réseaux sont articulés autour d'objets singuliers qui chacun possède, du fait de leur nature propre, une force, une efficacité plus ou moins grande. L'examen du bios de l'objet mémoriel implique de le considérer sous un angle plastique : il n'y a pas un objet ou un lieu de mémoire absolu qu'une histoire qui lui est extérieure polarise au gré des événements. Il importe au contraire, comme l'avaient bien pressenti les responsables politiques et militaires à la fin de la guerre, de prêter attention à la matérialité première des phénomènes de mémoire : car la mémoire n'est pas tant constituée autour de symboles, qu'incarnée dans des formes métonymiques, ou, plus exactement, le symbole se greffe sur des métonymies.

Plaque à l'entrée du Pavillon de Kannon pour l'Asie en marche

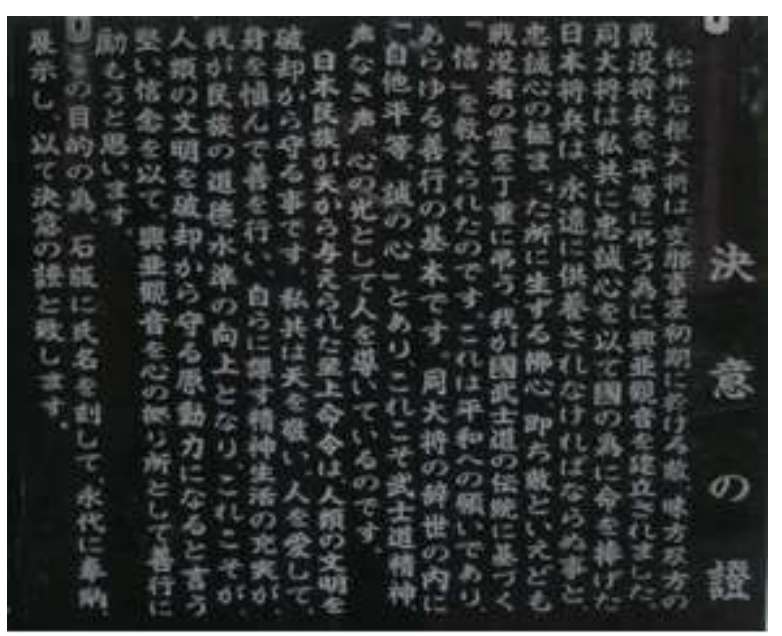

plaque À l'entrée du pavillon de kannon pour l'asie en marche (Kōa Kannon), Atami, 1939-1940

(C) $\mathrm{ML}$

3 Nous étudierons ici principalement les deux sites où sont conservés les restes des "criminels de guerre de catégorie A », le Pavillon de Kannon pour l'Asie en marche (Kōa Kannon-dō 興亜観音堂) à Atami et le Mausolée des sept Grands hommes morts pour la nation (Junkoku nanashi byō 殉国七士廟) sur le mont Sangane 三ケ根山 dans le département d'Aichi. Ces lieux, bien que peu connus du grand public japonais et étranger, sont très souvent mentionnés dans la littérature militante ou critique ${ }^{1}$. Nous nous appuierons sur ces différentes sources, ainsi que sur de nombreuses observations de terrain. Notre article sera découpé en deux grandes parties correspondant à deux grandes périodes : avant 1978 et après 1978, 1978 étant la date pivot à laquelle les sept personnes en question ont été enregistrées parmi les héros honorés au sanctuaire Yasukuni.

\section{Recueillir les restes pour préparer l'avenir}

\section{La fondation du Pavillon de Kannon pour l'Asie en marche}

4 Le Pavillon de Kannon (Avalokiteśvara) pour l'Asie en marche est situé à Atami, à une demi-heure à pied de la gare principale où passe le Shinkansen. Situé sur la baie de 
Sagami, il est, malgré sa proximité de la gare, relativement difficile d'accès, le chemin depuis la route étant raide et mal aménagé.

5 Toutefois, s'il est de petite taille et aujourd'hui mal indiqué, il a été conçu sous des auspices glorieux. Il fut en effet édifié sur les ordres du général Matsui Iwane 松井石根 (1878-1948), chef du Corps expéditionnaire de Shanghai d'août à décembre 1937, puis Commandant en chef des forces armées japonaises en Chine centrale jusqu'en mars 1938. Tenu pour responsable militaire des massacres perpétrés par les troupes japonaises à Nankin et dans les environs, il fut inculpé comme criminel de guerre de catégorie A et exécuté en 1948.

6 En mars 1938, suite aux protestations de la communauté internationale, Matsui avait été rappelé à Tōkyō et mis à la retraite ${ }^{2}$. Il décida alors la construction d'un temple sur le mont Narusawa-yama 鳴沢山 à flanc de coteau face à la mer, à proximité de la résidence secondaire qu'il venait de se faire construire. Ce temple était à ses yeux appelé à devenir, pour tous les morts de la guerre sino-japonaise, un peu l'équivalent bouddhique du sanctuaire Yasukuni, la figure de Kannon ayant été choisie car elle « transcende toutes les écoles» (kaku shūha ni chōetsu shite iru 各宗派に超越している)

7 Toutefois la spécificité de ce temple est que, suivant la volonté de Matsui, devaient y être honorées non seulement les victimes japonaises, mais aussi les victimes chinoises. C'est pourquoi à l'intérieur du temple furent placées une statuette de Kannon et, de part et d'autre, deux tablettes dorées, l'une au nom des "victimes japonaises des événements de Chine» (Shina jihen Nihon senbotsusha reii 支那事変日本戦没者霊位), l'autre au nom des «victimes chinoises des événements de Chine » (Shina jihen Chūka senbotsusha reii 支那事変中華戦没者霊位). Quelques tableaux vinrent décorer le lieu. $\mathrm{Au}$ mur, une œuvre du peintre nihonga Katayama Nanpū 堅山南風 (1887-1980) et, au plafond, une autre de Dōmoto Inshō 堂本印象 (1891-1975) figurant un dragon, symbole au Japon de la civilisation chinoise. Une copie du "Sūtra de Kannon $»^{4}$ calligraphiée par trente-trois bienfaiteurs du projet fut déposée dans le temple. Parmi les copistes, on trouve Konoe Fumimaro 近衛文麿 (1891-1945) et Suzuki Kantarō 鈴木貫太郎 (1867-1948), qui furent Premiers ministres de juin 1937 à janvier 1939 puis de juillet 1940 à octobre 1941 pour le premier, et d'avril à août 1945 pour le second, ainsi que les noms des supérieurs de plusieurs grands monastères représentant différentes écoles: le Kan.eiji, le Kōfukuji, le Zōjōji ou encore le Gokokuji5 ${ }^{5}$. Le petit bâtiment, achevé en février 1940, fut confié aux bons soins d'un moine issu de l'école Shingon, Itami Jinrei (ou Ninrai) 伊丹忍礼 ( ?-1985).

Parallèlement au temple, Matsui fit réaliser entre mars et décembre 1939 une statue à l'image du bodhisattva, incarnation de la compassion du bouddha. Il s'adressa pour cela à Shibayama Seifū 柴山清風 (1901-1969), potier et sculpteur de Tokoname 常滑 dans le département d'Aichi. Diplômé de l'École de céramique de la ville, ce dernier était depuis 1934 spécialisé dans la fabrication de statues de Kannon. Il en réalisa des milliers durant la période de la guerre, notamment de tout petits formats $(3 \mathrm{~cm})$ appelés Tamayoke Kannon 弾除け観音 ou Kannon pare-balles que l'on offrait comme amulette aux soldats. 

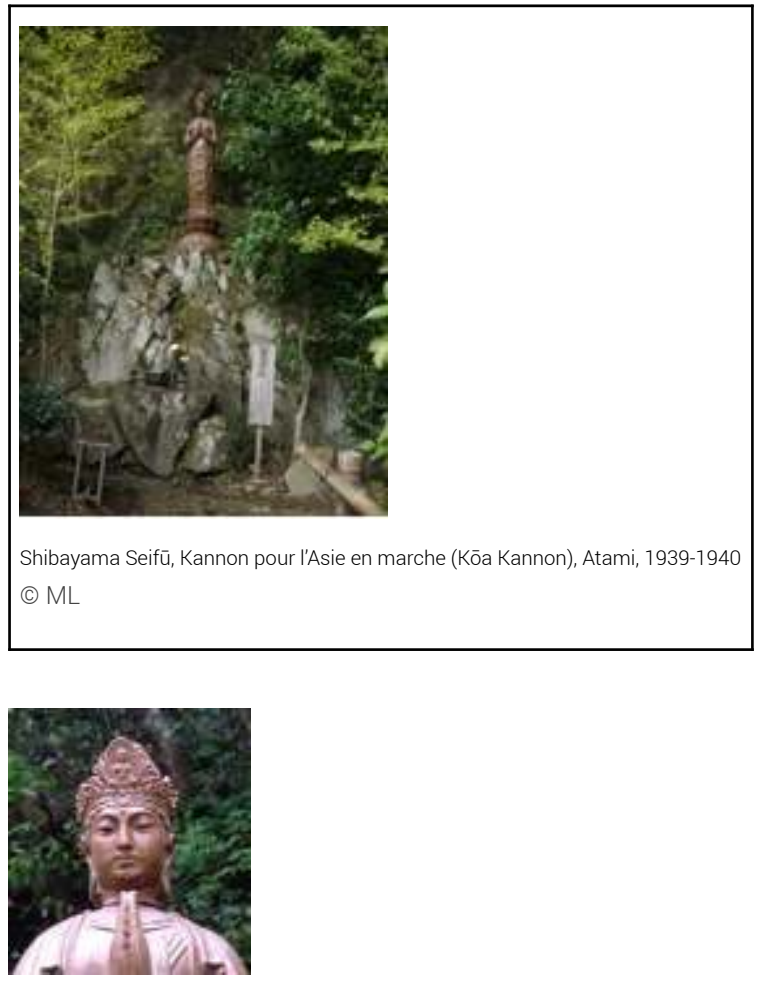

(détail)

(c) ML

Baptisée, d'après un des slogans de l'époque, Kannon pour l'Asie en marche et conçue pour être le vénéré principal du temple (honzon 本尊), la statue commandée par Matsui est une terre cuite émaillée haute de 3,3 $\mathrm{m}$, une taille remarquable pour une œuvre de ce type ${ }^{6}$. Dans le socle, en forme de fleur de lotus, est serrée une urne comprenant les cendres de certains soldats ayant servi sous ses ordres en Chine ${ }^{7}$. On notera qu'il n'est pas fait mention ici d'ossements provenant de soldats chinois.

Quand les travaux furent terminés, Matsui rédigea un petit texte de dédicace (engisho 縁起書), conservé sur place, récapitulant le sens qu'il donnait à son acte. Il en souligne, comme il est d'usage en telle occasion, la portée votive et propitiatoire :

Les événements de Chine sont des échanges de coups entre voisins et amis qui ont de façon ô combien tragique causé la perte d'un nombre considérable de vies. [...] Afin d'accompagner leurs mânes, nous édifions ici, avec de la terre ramenée de tous les champs de bataille du Jiāngnán et mêlée du sang versé par les nôtres comme par celui de nos ennemis, une statue du bodhisattva Kannon qui délivre de toute crainte et veille avec un œil de compassion sur tous les êtres; à travers sa vertu, nous rendons hommage pour l'éternité tant à ceux que l'on a aimés qu'à ceux que l'on a haïs, nous invoquons avec tous la puissance de Kannon, et prions pour que soit révélée la lumière éclatante de l'Asie de l'est.

支那事変は友燐相撃ちて莫大の生命を喪滅す、実に千載の悲惨事なり。(中略) 茲に此等の霊を中う為に、彼我の戦血に染みたる江南地方戦場の土を採り、施 無畏者意眼視衆生の観音菩薩の像を建立し、此の功徳を以て永く怨親平等に回 向し、諸人と俱に彼の観音力を念じ、東亜の大光明を仰がん事を祈る。

11 Toutefois, il ne faudrait pas surévaluer la dimension spirituelle ou compassionnelle du geste de Matsui. Il semblerait en effet que l'idée même d'édifier un temple lui fut soufflée par un aubergiste d'Atami, un certain Furushima Yasuji 古島安二, qui a fourni 
par ailleurs le terrain. Tanaka Masaaki 田中正明 (1911-2006), le secrétaire particulier de Matsui, rapporte d'ailleurs qu'à la proposition de Furushima, Matsui aurait répondu : «Faites ça, c'est une bonne idée! Mais sans déranger personne!»(ぜひやっ てく机。他人に迷惑をかけないでく机 $)^{8}$, à la suite de quoi la construction du temple fut entreprise par des associations locales d'anciens combattants et de jeunes, à partir d'un surplus de bois du Atsuta jingū 熱田神宮, le sanctuaire de Nagoya où est déposée l'épée Amenomurakumo no tsurugi 天叢雲剣 - l'un des trois regalia de l'institution impériale -, et qui avait à ce titre les faveurs de l'armée'. La fondation de ce temple doit donc être interprétée comme la rencontre des intérêts bien compris d'une communauté locale, désireuse de se distinguer en accueillant sur son sol la mémoire d'un des grands événements de l'époque, et d'un général soucieux d'asseoir sa place dans l'histoire et de laver l'opprobre pesant sur son nom après les remous des derniers mois de sa carrière. En effet, comme souvent dans les sphères du pouvoir, Matsui n'hésitait pas à utiliser l'art et la religion à des fins politiques : il commanda ainsi deux autres statues du même type à Shibayama, la première en 1941 pour l'offrir à Wāng Zhàomíng 汪兆銘 ${ }^{10}$, le chef pro-japonais du Gouvernement nationaliste de Nankin (Nánjīng guómín zhèngfŭ 南京國民政府), l'autre en 1943 pour Pibulsonggram, qui dirigeait alors la Thaïlande. Sa compassion à l'égard des victimes et notamment des victimes chinoises servait aussi l'image qu'il se faisait de lui et de son rôle dans l'histoire.

\section{La récupération des ossements}

12 Matsui Iwane, Tōjō Hideki 東條英機 (né en 1884), Doihara Kenji 土肥原賢二 (né en 1883), Hirota Kōki 広田弘毅 (né en 1878), Itagaki Seishirō 板坦征四郎 (né en 1885), Kimura Heitarō 木村兵太郎 (né en 1888), Mutō Akira 武藤章 (né en 1892) sont les sept plus hauts responsables japonais exécutés suite au Procès de Tōkyō. Tous étaient des généraux de l'Armée de terre, à l'exception de Hirota, Premier ministre de mars 1936 à février 1937, qui était un civil. Ils furent pendus à la prison de Sugamo et leurs dépouilles transportées à Yokohama pour y être incinérées. Dans un épisode bien connu, Sanmonji Shōhei 三文字正平 (1891-1975), l'avocat qui assurait la défense de Koiso Kuniaki 小僟國昭 (1880-1950), rapporte avoir dérobé dans la nuit du 26 décembre l'urne contenant pêle-mêle les cendres des sept condamnés à mort, et l'avoir secrètement confiée au supérieur du Kōzenji 興善寺, un temple situé dans le nord de Yokohama. Toutefois, quelques mois plus tard, en mai 1949, Sanmonji et quelques proches décidèrent de la déplacer et de la cacher à Atami dans le Pavillon de Kannon où avaient déjà été déposés les quelques restes de Matsui confiés par les Américains à sa famille : une paire de lunettes, une couronne dentaire, des poils de barbe. À compter de cet instant, le Pavillon de Kannon prit une autre dimension. Il devenait le dépositaire non seulement du souvenir de Matsui et de la campagne de Chine, mais des sept condamnés à mort et à travers eux de toute la mémoire de la défaite.

Cet épisode fut déterminant, car l'Occupation fut la seule période où le cours de l'histoire put être radicalement changé. En n'inculpant pas l'Empereur et la famille impériale, en permettant que soient récupérés les restes des principaux criminels de guerre, et en menant une campagne très incomplète d'éradication des monuments et symboles du militarisme impérial ${ }^{11}$ - ce qui fait par exemple qu'il reste encore à travers l'archipel des dizaines de petits monuments portant une inscription de la main de Tōjō Hideki -, l'administration de MacArthur (ou SCAP) ${ }^{12}$ a évidemment une responsabilité 
importante quant à toute la politique mémorielle qui s'est développée après 1952. Même s'il faut éviter le paradoxe qui veut qu'on reproche tantôt aux Américains d'avoir trop censuré, tantôt de ne pas l'avoir assez fait, il est nécessaire de rappeler cette évidence, qu'on évoque la question du Yasukuni ou celle des manuels scolaires.

La signature du Traité de San Francisco fut suivie d'un violent mouvement de réaction visant à réhabiliter l'action du pays pendant la guerre. Alimenté par le retour aux affaires de hautes personnalités de l'époque, ce mouvement se manifesta concrètement : par l'organisation de célébrations au Yasukuni et dans les Sanctuaires pour la protection du pays (Gokoku jinja 護国神社), comme celle du 18 octobre 1951 en présence du Premier ministre Yoshida Shigeru; par des publications tendant à légitimer le conflit et ses responsables, à commencer par le déferlement en 1952-1953 de recueils de textes de criminels de guerre ${ }^{13}$; par des autorisations données à des associations comme la Hakuō izoku-kai 白鴎遺族会 (Association des familles de victimes Les Mouettes blanches) en $1952^{14}$ dont le but était de défendre l'héroïsme des kamikazes en réaction à la lecture pacifiste des éditeurs du célèbre recueil Kike wadatsumi no koe きけわだつみのこえ (Ecoutez les voix des esprits de la mer! 1949) ${ }^{15}$. Enfin, ce phénomène fut rendu visible dans l'espace de la nation par la construction d'un certain nombre de monuments, notamment un autre Pavillon de Kannon, inauguré en 1955 à proximité de l'ancienne base aérienne de Chiran 知覧, au sud de Kyūshū, d'où partirent nombre de kamikazes, ou encore la statue L'Amour ( $A i$ 愛), construite la même année en face de la gare de Tōkyō par Yokoe Yoshizumi 横江嘉純 (1887-1962), en mémoire des criminels de guerre ${ }^{16}$.

L'Amour

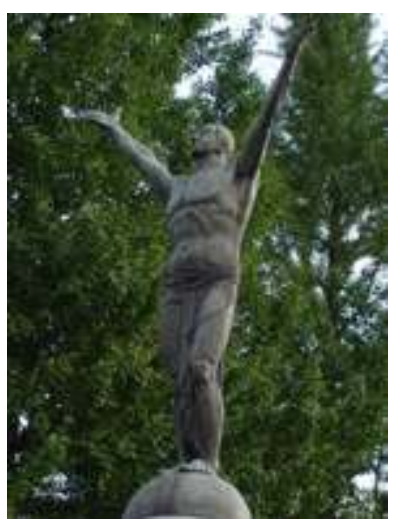

Yokoe Yoshizumi, L'Amour, Tōkyō, 1955

(c) $\mathrm{ML}$

C'est dans ce contexte réactionnaire, qui est aussi celui des débuts de la Guerre froide, que fut érigée en avril 1959 dans le temple d'Atami une grande stèle servant de pierre tombale à Matsui, Tōjō, Doihara, Hirota, Itagaki, Kimura et Mutō, à côté de laquelle fut placée une autre stèle à la mémoire des 1054 criminels de guerre de catégories $B$ et $C$ exécutés durant l'occupation. Taillée dans la pierre brute, elle porte en son milieu un glyphe facile à décoder vu le lieu : «Stèle des sept Grands hommes » (Nanashi no hi 七士 之碑), gravée en suivant une calligraphie demandée spécialement pour l'occasion - on parle en japonais d'un kigō 揮毫 ${ }^{17}$ - à Yoshida Shigeru 吉田茂 (1878-1967), Premier ministre de 1948 à 1954, ce qui est au minimum, vu le caractère héroïsant de cette 
inscription, l'expression d'une certaine sympathie de sa part à l'égard des responsables de la guerre exécutés alors qu'il était en fonction.

Stèle des sept Grands hommes

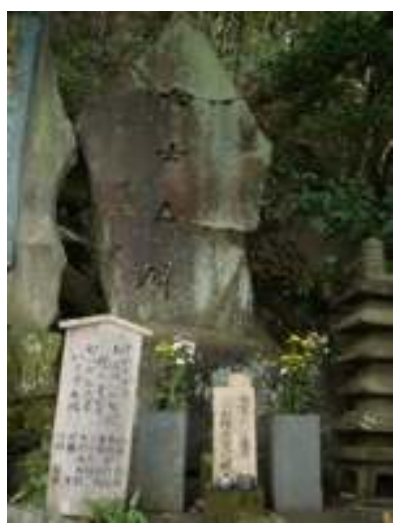

Stèle des sept Grands hommes (Nanashi no hi), Atami, 1959

(c) ML

D'ailleurs, plusieurs Premiers ministres répondront positivement à des sollicitations similaires. Kishi Nobusuke 岸信介 (1896-1987) cautionna par exemple en 1984 le Mausolée des sept Grands hommes dont les piles d'entrée sont gravées d'après sa main, et Koizumi Jun.ichirō 小泉純一郎 (1942-) fit de même en 2004 pour une stèle à l'entrée du Centre pour la paix des forces d'assaut spéciales (i.e. les kamikazes) (Tokkō heiwa kaikan 特攻平和会館) de Chiran. Chacun de ces hauts responsables était du reste présent lors de l'inauguration du monument qu'il avait ainsi honoré. Alors que le pouvoir politique est prisonnier de l'article 11 du Traité de San Francisco qui prévoit que le «Japon accepte les décisions du Tribunal militaire international pour l'ExtrêmeOrient », les responsables des partis conservateurs au pouvoir (Jiyū-tō 自由党 puis Jimin-tō 自民党 ou PLD), en permettant que leur soutien soit littéralement scellé dans la pierre, ont dès les années 1950 non seulement affirmé la nécessité d'une réhabilitation de l'action de leurs pairs ou aînés pendant la guerre, reniant l'esprit de leurs engagements internationaux ${ }^{18}$, mais aussi contraint leurs compatriotes à gérer ce problème après eux car on sait à quel point il est difficile de supprimer un monument, a fortiori lorsqu'il porte le nom d'un chef de gouvernement. On peut même soutenir que l'intérêt récent des historiens et du public à l'égard des monuments liés à la guerre n'est jamais que la conséquence de cette politique de réorientation mémorielle initiée dans les années 1950.

Piles d'entrée du Mausolée des sept Grands hommes morts pour la nation 


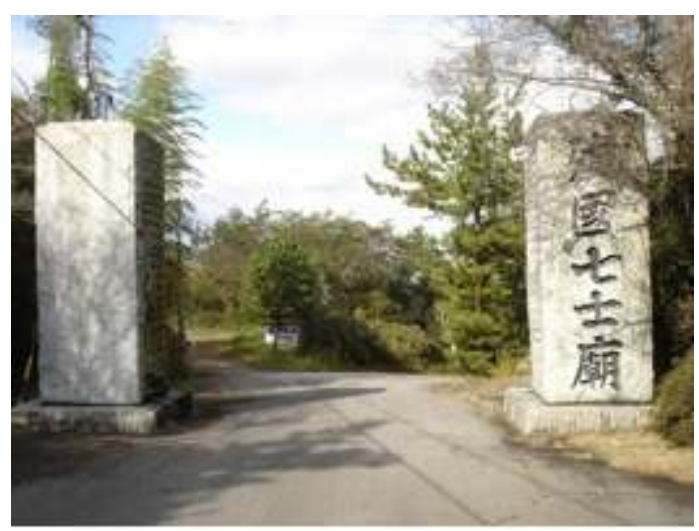

Piles d'entrée du Mausolée des sept Grands hommes morts pour la nation (Junkoku nanashi-byō), mont Sangane, 1984

(c) $\mathrm{ML}$

Stèle à l'entrée du Centre pour la paix des forces d'assaut spéciales

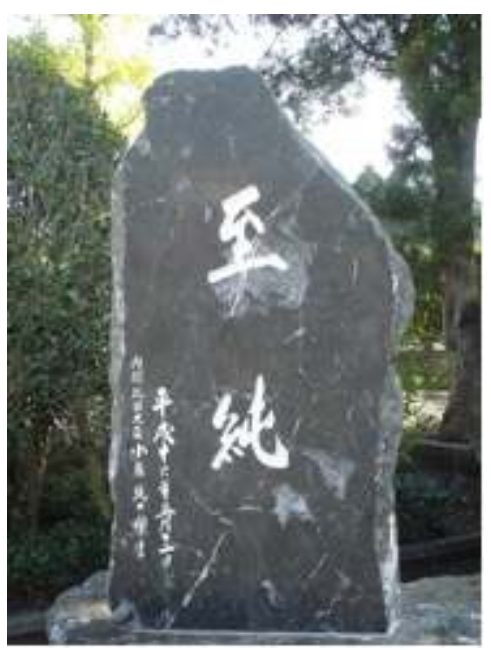

Stèle à l'entrée du Centre pour la paix des forces d'assaut spéciales (Tokkō heiwa kaikan), Chiran, 2004

(C) $\mathrm{ML}$

17 Le Pavillon de Kannon pour l'Asie en marche fut la première sépulture des sept criminels de guerre de catégorie A exécutés. Néanmoins, dès la fin de l'occupation, Sanmonji et les autres avocats des inculpés du Procès de Tōkyō ont eu en tête de faire construire un monument qui leur serait spécialement dédié, le temple d'Atami étant très marqué par Matsui et son histoire personnelle. Il fut alors décidé de récupérer une partie des ossements (bunkotsu 分骨) et de les enterrer au sein d'un mausolée construit pour l'occasion ${ }^{19}$. Toutefois, c'est là encore la figure de Matsui Iwane qui servit de fil directeur puisque l'endroit qui fut choisi, sur le mont Sangane au nord de la ville de Gamagōri 蒲郡, se trouve dans le département d'Aichi dont ce dernier était originaire ${ }^{20}$. Inauguré le 16 août 1960, en présence des familles, d'anciens militaires et de hauts responsables politiques, le Mausolée comprend un petit tertre que domine une tombe en granit rose à cinq étages terminée par une stèle portant l'épigraphe «Tombe des sept Grands hommes » (Junkoku nanashi haka 殉国七士墓) ${ }^{21}$. 


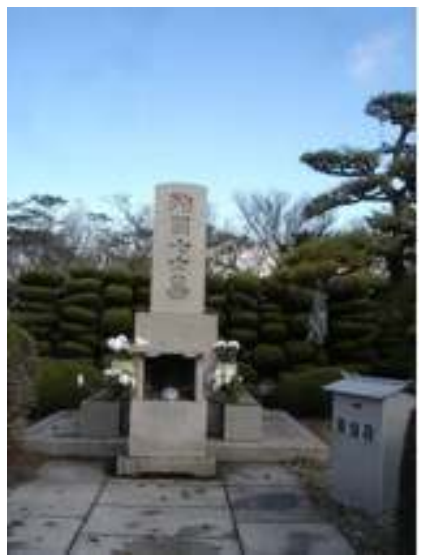

Tombe des sept Grands hommes (Junkoku nanashi haka), mont Sangane (c) ML.

Plaque à côté de la Tombe des sept Grands hommes

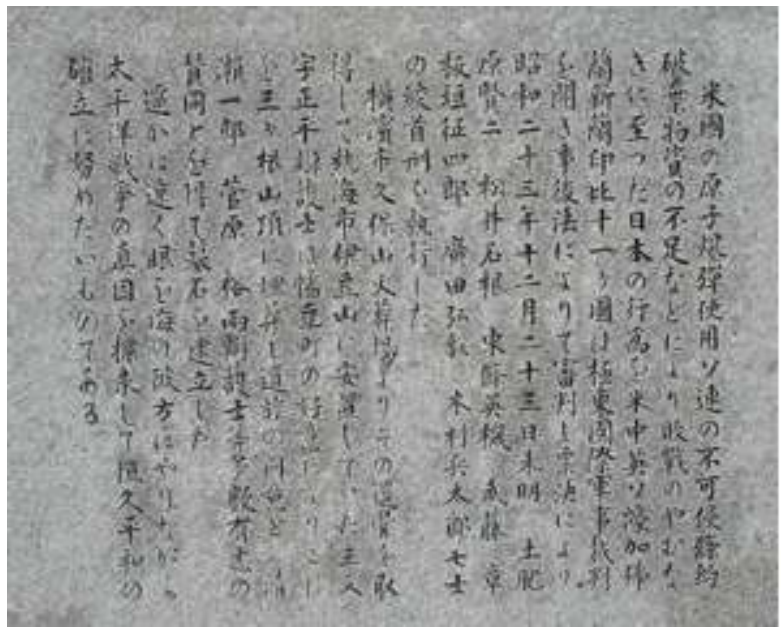

Plaque à côté de la Tombe des sept Grands hommes (Junkoku nanashi haka), mont Sangane (c) ML

18 Cette inscription fut tracée d'après un modèle d'Araki Sadao 荒木貞夫 (1877-1966), général de l'Armée de terre condamné à la prison à perpétuité en 1948, libéré en 1955, dont le monument commémoratif construit en 1967 à Totsukawa 十津川 sera à son tour gravé d'après un kigō de Satō Eisaku 佐藤栄作 (1901-1975), alors Premier ministre.

Ces deux monuments ne sont pas les seuls à transmettre le souvenir de ces hommes. A l'instar de la statue en face de la gare de Tōkyō mentionnée précédemment, d'autres ont été érigés à leur mémoire à peu près à la même période ${ }^{22}$. Par exemple, en 1968, une stèle fut construite à Yokohama, à proximité du crématorium où ont été traités les corps des criminels de guerre.

Portant l'épigraphe "Stèle pour les âmes fidèles des soixante preux " (Rokujū resshi chūkon-hi) 六十烈士忠魂碑, elle a été construite pour, d'après le texte inscrit sur le côté, « les soixante personnes, à commencer par leur chef, le Premier ministre Tōjō, qui 
ont été incinérées en cet endroit sur le mont Kuboyama après avoir été exécutées à la prison de Sugamo suite aux procès iniques du Tribunal militaire d'Extrême-Orient » 不 法な極東軍事裁判の結果、巣鴨刑務所で極刑死せられ、当地久保山で荼毘に付さ れた東条首相以下六十名. Il existait par conséquent avant même 1970 plusieurs lieux commémoratifs dédiés aux criminels de guerre de catégorie A exécutés, ou bien les incluant, auxquels il faudrait encore ajouter les cénotaphes individuels (ou les caveaux), dans lesquels les familles, à défaut d'ossements, ont pu placer des objets personnels ou une mèche de cheveux. Pour information, celui de Tōjō se trouve au cimetière de Zoshigaya 雑司ヶ谷 (Tōkyō), celui de Doihara au cimetière du Gokokuji (Tōkyō), celui de Hirota au cimetière du Shōfukuji 聖福寺 (Fukuoka), celui de Kimura au cimetière d'Aoyama (Tōkyō).

21 Néanmoins, le Temple d'Atami et le Mausolée du mont Sangane occupent, en raison de la présence de cendres, une place à part. Le premier s'inscrit dans le cadre du bouddhisme, le second peut être considéré comme laïc et public, d'autant que le terrain où il se trouve a été mis à disposition par la commune de Hazu 幡豆 et que le mont Sangane fait partie du Parc national de la baie de Mikawa (Mikawa-wan kokutei kōen 三 河湾国定公園 $)^{23}$. Quand on considère l'histoire de la commémoration des morts de la nation dans le Japon d'après-guerre, où l'on retrouve quasiment toujours le triptyque " commémoration bouddhique / shintō / publique ", il ne manquait pour achever de mettre ces hommes sur un pied d'égalité avec tous les autres morts de la nation que leur enregistrement au Yasukuni. Cet enregistrement qui interviendra en octobre 1978 est donc l'aboutissement d'un long et patient processus dans lequel, à défaut de l'État, se sont, à la suite de Yoshida, investis publiquement et à long terme plusieurs des principaux dirigeants du pays. Il ne faut donc pas considérer leur inscription dans les registres du Yasukuni comme un "premier geste », comme le «minimum » que la nation devait faire pour, trente ans après leur exécution, leur rendre malgré tout un petit hommage et apaiser les mémoires, ainsi qu'on le soutient parfois. C'est au contraire une étape relativement avancée au sein d'une stratégie mémorielle initiée bien en amont visant à créer les conditions pour un renversement des grilles historiographiques imposées par la défaite et le Procès de Tōkyō.

\section{Vers la révision historique}

\section{Le tournant de 1978}

Le 12 décembre 1971, un attentat à l'explosif fut perpétré par les membres du futur Front armé anti-japonais de l'Asie orientale (Higashi-Ajia hannichi busō sensen 東アジ ア反日武装戦線) à l'encontre du Pavillon de Kannon et de la Stèle des sept Grands hommes d'Atami ${ }^{24}$. La stèle fut brisée, mais le temple resta intact, la charge qui devait le détruire n'ayant pas fonctionné (on notera que la stèle put être réassemblée). Cet épisode, qui fut le premier d'une série, montre que les mouvements d'extrême-gauche percevaient l'importance physique et symbolique de ce site. Toutefois le groupe en question étant encore inconnu ${ }^{25}$, l'affaire fit relativement peu de bruit. Au final, il semblerait même qu'elle ait surtout contribué, du fait de l'indignation soulevée, à normaliser l'existence du lieu.

L'enregistrement au Yasukuni des quatorze criminels de guerre de catégorie A morts pendant l'occupation (les 7 qui furent exécutés et les 7 qui moururent en captivité) ${ }^{26}$ 
eut lieu en octobre 1978, deux mois après la signature, encore parfois jusque dans des publications scientifiques ${ }^{27}$, aucun reste biologique n'est conservé le 12 août, du Traité de paix et d'amitié sino-japonais. Les cendres de Matsui, Tōjō et des autres ne furent cependant pas déplacées à cette occasion, puisque, contrairement à ce qu'on lit dans le sanctuaire. En revanche, suivant les croyances shintō, leurs mânes y sont solidairement réunies avec toutes celles des autres défunts sans qu'il soit possible, selon les responsables du Yasukuni, de les en extraire ${ }^{28}$.

La consécration des criminels de guerre dans le célèbre sanctuaire eut pour effet d'enclencher un long processus de confrontation mémorielle entre la Chine et la Corée d'une part et le Japon de l'autre. Côté chinois, elle s'exprima, entre autres, par l'ouverture en 1987 du Musée mémorial de la guerre anti-japonaise du peuple chinois (Zhōngguó rénmín kàngrì zhànzhēng jìniànguăn 中国人民抗日戦争記念館), où même s'il est légitime de présenter le Japon comme un agresseur extrêmement brutal -, tout ce que les rivalités internes ont pu alors engendrer de drames tend à être occulté de l'histoire au profit de l'unité nationale. Côté japonais, les événements majeurs de cette crispation autour de la mémorialisation de la guerre sont les visites officielles du Premier ministre Nakasone Yasuhiro 中兽根康弘 (1918-) au Yasukuni en 1984-1985 et la publication des manuels d'histoire révisionnistes de la Tsukuru-kai en 2001 et $2005^{29}$.

Toutefois, les trente dernières années ont aussi vu se multiplier des actions moins visibles, mais dont la portée est loin d'être négligeable. Depuis le début des années 1970 , et surtout après 1978 , se sont ainsi développées les constructions ou les rénovations de monuments commémorant l'action des forces japonaises dans la guerre. Sur le mont Sangane, par exemple, ont été construits, en contrebas de la Tombe des sept Grands hommes, seize monuments dédiés à différents bataillons ou régiments. Or, si le plus ancien remonte à 1963 , la plupart sont postérieurs à 1978 et le plus récent date de 1987. Les piles d'entrée du Mausolée, mentionnées précédemment, ainsi que la plaque en marbre expliquant la raison d'être du site datent de 1984. Idem pour un grand monument érigé en face du parking représentant une flamme et portant l'épigraphe «Paix éternelle» (Bansei taihei 万世太平). Autrement dit, ce mémorial n'a pas seulement été entretenu, il a considérablement grossi et sa nature est expliquée aux visiteurs beaucoup plus clairement qu'elle ne l'était autrefois. Il est donc davantage visité et peut distiller son message avec d'autant plus de force.

De façon plus générale, c'est tout le matériel commémoratif lié aux soldats morts pendant la Seconde Guerre mondiale qui s'est développé pendant cette période. Il n'est qu'à se rendre dans les sanctuaires départementaux pour la protection du pays : que ce soit à Nagoya, Ōsaka, Kyōto, on remarque systématiquement des stèles qui datent de la fin des années 1970 ou des années 1980.

Stèles à droite du Sanctuaire du mont Ryōzen pour la protection du pays 


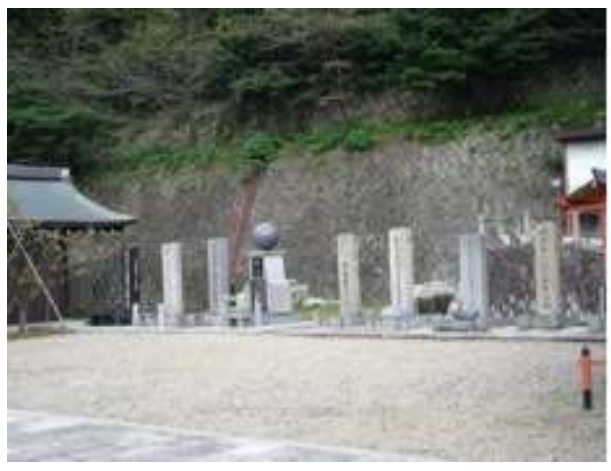

Stèles à droite du Sanctuaire du mont Ryōzen pour la protection du pays (Ryōzen gokoku jinja) (c) ML

Certaines mentionnent même explicitement le nom de criminels de guerre de catégorie A, comme la «Stèle à la gloire des âmes des valeureux guerriers martyrs exécutés par décision de justice » (Hōmushi junkoku resshi irei kenshō hi 法務死殉国烈士慰霊顕彰 碑) du sanctuaire Kibitsu 吉備津神社, à Okayama, où figure le nom de Doihara Kenji. De même, on peut citer le cas du parc commémoratif qui entoure la statue Kannon des Philippines (Hitō Kannon 比島観音), à un kilomètre environ du Mausolée des sept Grands hommes, sur le mont Sangane. Tout autour de cette statue en bronze réalisée en $1972^{30}$, se trouve une centaine de monuments dédiés principalement à des unités ayant servi aux Philippines.

\section{Parc de Kannon des Philippines}

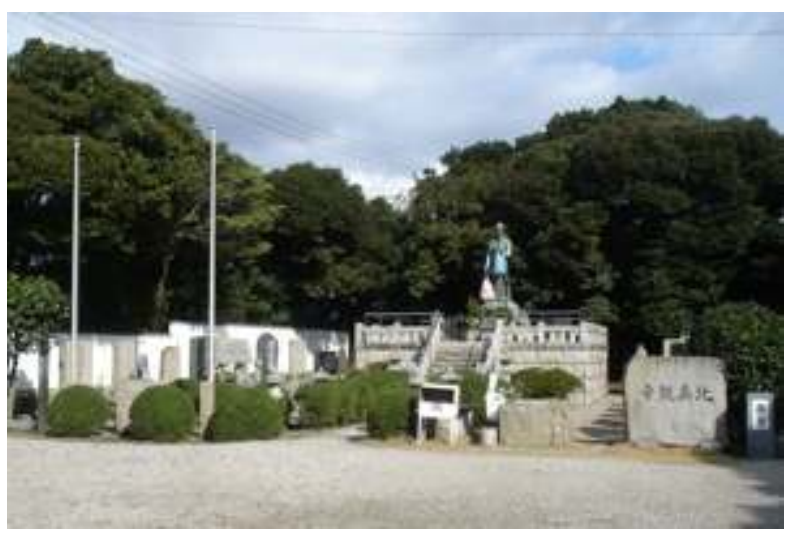

Parc de Kannon des Philippines (Hitō Kannon), mont Sangane

(c) $\mathrm{ML}$

Tous ne sont pas datés, mais tous sont postérieurs à 1972. Et les derniers ont été construits dans les années 2000, le grand panneau à l'entrée, portant la mention 1999 et donnant le plan du site, n'étant manifestement pas à jour. Ce parc commémoratif, qui, par le nombre de monuments rassemblés, est l'un des plus importants du pays, est donc non seulement récent, mais encore en développement.

Cette évolution nous rappelle que l'historien ne peut pas se contenter de s'interroger sur la genèse des monuments et sur leur dimension symbolique, il doit s'intéresser à ce qu'ils deviennent, à leur insertion dans un milieu, à l'effet qu'ils dégagent, à leur nature plastique, c'est-à-dire à leur capacité à engendrer de l'histoire. Ceci est d'autant plus 
important que l'historien est souvent lui-même un produit du monument, tout comme il est un produit du document, dans la mesure où sa rencontre avec l'objet a éveillé sa curiosité et orienté son travail.

\section{L'Association pour la sauvegarde du Pavillon de Kannon pour l'Asie en marche}

Quand on se rend aujourd'hui à Atami au Pavillon de Kannon pour l'Asie en marche, on est frappé par le caractère modeste et désolé du lieu. On peut facilement imaginer, suivant les dires de Mlle Itami, l'une des trois filles d'Itami Jinrei qui s'occupent aujourd'hui du temple, des jours entiers sans aucun visiteur. Il n'y a pas de parking, ni distributeur de boissons ou toilettes comme partout dans l'archipel. Malgré la nature historique du lieu et la beauté du site, ce n'est pas un endroit touristique. Seule en bas de la rampe, une bannière signale que le temple fait partie d'un circuit organisé.

L'endroit n'est pas pour autant abandonné. Comme dans tous les lieux de ce type, il existe une association de fidèles (hōsankai) qui organise les manifestations autres que les récitations, prières ou offrandes quotidiennes. Depuis la fin de l'occupation, cette association propose à dates régulières des célébrations publiques: les jours les plus couramment retenus ont tous une portée symbolique, qu'il s'agisse du 3 mars, ancien Jour de l'Armée de terre (Rikugun kinenbi), du 15 août, date de la fin de la guerre, des alentours du 18 mai et du 18 octobre, dates des célébrations de printemps et d'automne au Yasukuni, et surtout du 23 décembre, anniversaire des exécutions qui coïncide merveilleusement aux yeux des intéressés avec celui de l'empereur Akihito. A ces occasions, se rassemblent quelques dizaines, voire quelques centaines de personnes au maximum.

Toutefois, au début des années 1990, se fit sentir le besoin de constituer une nouvelle association, plus large et davantage tournée vers les questions politiques et idéologiques. Les premières discussions sérieuses sur ce projet eurent lieu fin 1992. Elles se concrétisèrent par une réunion à l'automne suivant au Kudan kaikan, hall de réunions et de conférences situé à deux pas du Yasukuni qui était jusqu'à la fin de la guerre le Grand cercle des officiers (Gunjin kaikan). L'association vit le jour encore un an plus tard, en novembre 1994, et prit le nom d'Association pour la protection de Kannon pour l'Asie en marche (Kōa Kannon wo mamoru kai).

Les principaux fondateurs de cette association sont Tanaka Masaaki (président), Tokutomi Tasaburō 徳富太三郎 (1925-), petit-fils de Tokutomi Sohō 徳富蘇峰 (lequel fut pendant la guerre un proche de Matsui Iwane), Odamura Shirō 小田村四郎 (1923-), ancien haut fonctionnaire, dirigeant des éditions Meisei-sha 明成社 et figure de la droite conservatrice, Nagoshi Futaranosuke 名越二荒之助 (1923-), intellectuel de droite et grande figure des mouvements révisionnistes, Matsudaira Nagayoshi 松平永 芳 (1915-2005), desservant principal du Yasukuni entre 1978 et 1992 qui prit la responsabilité d'inclure les criminels de guerre, ou encore Itagaki Masashi 板坦正 (1924-), fils cadet d'Itagaki Seishirō et élu de la Chambre haute entre 1980 et 1997. Toutefois, bien que cette association revendique aujourd'hui 2300 membres environ ${ }^{31}$, son importance ne doit pas être surestimée. Il y a en effet de nombreux groupes d'extrême-droite nationalistes au Japon, et la plupart des membres nommés ci-dessus appartiennent aussi à d'autres mouvements. Toutefois, si on la considère dans le long processus décrit précédemment - des premières tentatives de Matsui de placer les 
massacres de Nankin sous le signe de la fatalité, jusqu'à l'enregistrement des criminels de guerre de catégorie A au sein du Yasukuni -, elle prend une dimension particulière. Il est par ailleurs intéressant de noter que sa création coïncide précisément avec «la contre-attaque néo-nationaliste commencée en 1993 [et qui] s'est poursuivie vers 1996 avec la fondation du Tsukuru-kai et des autres sociétés concernées par l'enseignement de l'histoire $»^{32}$. En effet, les principaux membres de l'Association pour la protection de Kannon, comme on peut le voir ci-dessus, sont de la génération de Taishō : autrement dit, la « contre-attaque néo-nationaliste » que décrit Lavelle peut aussi être interprétée comme le fruit du dernier effort des acteurs même de la guerre pour transmettre le flambeau d'un demi-siècle d'opposition aux conséquences de la défaite. Même si des facteurs idéologiques et conjoncturels peuvent expliquer le raidissement des néonationalistes dans les années 1990, chronologiquement, on observe qu'il n'y a pas de rupture entre leur action et celle des derniers survivants du militarisme.

Ce point est d'autant plus net que la question historiographique en général figure au cœur des préoccupations de l'association, comme on peut le voir dans le premier numéro de leur bulletin, en 1994, où sont résumés comme suit leurs trois principaux objectifs :

- Apaiser les âmes des victimes de guerre (les condamnés à mort compris) 戦死者(死刑者も含 む)の鎮魂

- Être blanchis du soi-disant Grand massacre de Nankin いわゆる「南京大虐殺」の雪冤

- Réviser la vision de l'histoire propagée par le Procès de Tōkyō 東京裁判史観の是正 ${ }^{33}$

Toutefois, à lire ce bulletin, on comprend vite que le nœud historique n'est pour eux ni la guerre du Pacifique, ni les bombardements américains de 1945, ni l'occidentalisation de Meiji : c'est l'occupation de la Chine et les massacres de Nankin qui constituent la question fondamentale. La majorité des articles de fond reviennent sur ce problème. Certes les arguments sont souvent spécieux voire révoltants, comme lorsque Tanaka défend l'amour de Matsui pour la Chine en expliquant que ce dernier avait recueilli une petite chinoise orpheline ${ }^{34}$. En revanche, quand on prend du recul, on ne peut qu'être frappé d'une chose: de 1939, avec la création du temple d'Atami, jusqu'au début des années 2000 avec ce bulletin, un groupe d'hommes appartenant aux plus hautes sphères de l'armée et du pouvoir, impliquant ouvertement la plupart des Premiers ministres, de Konoe à Nakasone, a tenté de justifier sa guerre sur le continent, épisode dont il fait la clé de voûte de la Seconde Guerre mondiale et autour duquel il a construit patiemment tout un système commémoratif. A ce phénomène deux causes se mêlent dans des proportions que nous n'essaierons pas d'évaluer: la première est un traumatisme psychologique lié au sentiment d'injustice que ces hommes ont ressenti quant au sens donné à leur combat en Asie dans les années 1930 ; la seconde, comme nous l'avons esquissé en introduction du Japon après la guerre, est une instrumentalisation de la mémoire, dont le but aura été la conservation du pouvoir ${ }^{35}$. Quoi qu'il en soit, la profondeur historique du système commémoratif qu'ils ont mis sur pied et son ancrage physique dans le sol de la nation nous laissent penser qu'il sera extrêmement difficile de le faire disparaitre ou d'en inverser d'un coup la polarité.

\section{Conclusion}

Même si le sanctuaire Yasukuni devait un jour, dans un revirement complet, accepter de retirer les noms des criminels de guerre de ses registres, ou si un mémorial public 
était construit, cela ne changerait rien au fait qu'un maillage de pierres, d'ossements et de reliques transmet à travers tout l'archipel une vision héroïque de l'action des principaux responsables du militarisme impérial et tend à maintenir le Japon dans un régime d'historicité d'« après-guerre ».

Toutefois notre but n'est pas tant ici de dénoncer une situation que d'énoncer un constat, à partir duquel proposer à chacun de développer un rapport réactif et dynamique aux objets mémoriels. En l'occurrence, ne pas se laisser méduser par la solennité de la pierre, éviter de prendre les monuments au sérieux, parce que c'est justement ce qu'ils veulent et ce que, à travers eux, veulent ceux qui les ont commandés. Toutefois l'exercice est difficile car les qualités plastiques des monuments - la dureté, la fixité - ont justement pour but de contraindre le passant à se soumettre à leur vérité.

Ceci est d'autant plus vrai que, comme nous l'évoquions en introduction, les objets mémoriels fonctionnent en réseau. Dans un précédent article sur le sort des statues durant l'immédiat après-guerre, nous avions mis l'accent sur les répercussions en termes d'occupation de l'espace public de la politique mémorielle ${ }^{36}$. C'est encore le cas ici, quoi que cela ne se traduise pas en termes d'urbanisme. On aura noté en effet que les principaux sites que nous avons mentionnés sont tous situés sur ou à proximité immédiate de l'épine dorsale du Japon qu'est le Tōkaidō et sa ligne de train à grande vitesse. De la gare de Tōkyō au mont Sangane, en passant par Yokohama ou Atami, on pourrait visiter l'ensemble en une seule journée. Ce point est évidemment symbolique de l'importance qu'on a voulu donner à ces monuments. Mais on pourrait aussi l'interpréter comme la capacité des monuments à s'organiser d'eux-mêmes, à créer des circulations, quand bien même - et c'est le cas ici - leur existence aurait quelque chose de souterrain. Les formes contraignent le regard, produisent du discours, réorganisent les mémoires, agissent sur les sociétés, mais elles prévoient aussi des déplacements, exerçant leurs forces jusque sur les corps.

\section{NOTES}

1. Parmi les ouvrages récents, voir: Sven Saaler, Politics, Memory and Public Opinion: the History, Textbook Controversy and Japanese Society, München, Iudicium, 2005; Franziska Seraphim, War Memory and Social Politics in Japan, 1945-2005, Harvard University Asia Center, 2006; Takahashi Tetsuya 高橋哲哉, Yasukuni mondai, Tōkyō, Chikuma shoten, coll. «Chikuma shinsho », 2005. Il n'existe toutefois quasiment aucune étude spécialisée sur le sujet; voir néanmoins Katō Nobuyuki 加藤信行, «Senpan no bohi wo sasaeru hitobito: Kōa Kannon - Junkoku nanashi no hi wo megutte », Shūkyō kenkyū, Nihon shūkyō gakkai, mars 2007, 159-160.

2. Matsui Iwane continua néanmoins d'occuper différentes fonctions hors de l'armée, notamment celle de Conseiller du gouvernement (Naikaku sangi) jusqu'en janvier 1940.

3. Matsui Iwane, « Kōa Kannon no buka eirei ni sasagu », Shufu no tomo, Tōkyō, avril 1940.

4. Le «Sūtra de Kannon » est un chapitre du Sūtra du Lotus (chap. 25 dans la traduction de JeanNoël Robert, Fayard, 1997). 
5. Tanaka Masaaki, «Dare yori mo Chūgoku wo ai shita hito : Matsui Iwane », Kōa Kannon, n 1 , automne 1994. Ce bulletin, dont il existe aussi une version papier, est accessible en ligne sur le portail www.history.gr.jp (février 2009).

6. «Seifū no tōbō Fukujukai », Asahi shinbun, juillet et novembre 1939.

7. Matsui Iwane, « Kōa Kannon no buka eirei ni sasagu », op. cit.

8. Tanaka Masaaki, « Dare yori mo Chūgoku wo ai shita hito : Matsui Iwane », op. cit.

9. Itami Jinrei, « Kōa Kannon wa dō shite konryū sareta ka », Kōa Kannon, n 3, 18 octobre 1995.

10. «Ōshi e Kōa Kannon-butsu », Yomiuri shinbun, 25 juin 1941.

11. Cf. Michael Lucken, «Le sort des statues et monuments commémoratifs durant l'occupation américaine ", Cipango, $n^{\circ} 14,2007$, p. 128-165.

12. L'acronyme SCAP (Supreme Commander of the Allied Powers) est à l'origine le titre même de MacArthur. Par extension, il désigne aussi son administration.

13. Plus d'une quinzaine de recueils de lettres et autres textes de criminels de guerre ou d'inculpés furent publiés entre avril 1952 et fin 1953, parmi lesquels : Shi shite sokoku ni ikin : shi senpan shikeishū no isho 死して祖国に生きん-四戦犯死刑囚の遺書, Tōkyō, Sōju-sha, 1952; Asu no asa no 'kuji': Daitōa sensō de sensō hanzaisha toshite shokei sareta hitobito no isho-shū あすの朝の 「九時」一大東亜戦争で戦争犯罪者として処刑された人々の遺書集, Tōkyō, Nihon shūhō-sha, 1952 ; Okada Tasuku 岡田資, Sugamo no jūsan kaidan : senpan shokeisha no kiroku 巣鴨の十三階段戦犯処刑者の記録, Tōkyō, Atō shobō, 1952; Ware shinu beshi ya: BC-kyū senpansha no kiroku われ 死ぬべしや-BC 級戦犯者の記録, Tōkyō, Atō shobō, 1952; Sokoku e no isho: senpan shikeishū no shuki 祖国への遺書-戦犯死刑囚の手記, Tōkyō, Mainichi shinbun-sha, 1952; Are kara shichinen: gakuto senpan no gokuchū kara no tegami あ孔から七年-学徒戦犯の獄中からの手紙, Tōkyō, Kōbunsha, 1953 ; Seiki no isho 世紀の遺書, Tōkyō, Sugamo isho hensankai kankō jimusho, 1953.

14. Le recueil publié par la Hakuō izoku-kai est intitulé Au bout du défilé des nuages (Kumo nagaruru hate ni 雲ながるる果てに), Tōkyō, Nihon shuppan kyōdō kabushiki gaisha, 1952.

15. Ce recueil (Kike wadatsumi no koe, Tôkyô daigaku kyôdô kumiai shuppanbu, 1949) fut publié par une association d'étudiants pacifistes de l'Université de Tōkyō. Grand succès de librairie, plusieurs éditions ont été diffusées jusqu'à récemment. Sur les répercussions de ce mouvement, voir Franziska Seraphim, op. cit, p. 135-158.

16. Cette statue fut commanditée par l'Association pour la compilation des lettres des victimes de Sugamo (Sugamo isho hensan-kai 巣鴨遺書編纂会) dont le recueil, mentionné ci-dessus, Seiki no isho 世紀の遺書 (Le testament du siècle), fut un important succès. Elle fut inaugurée en 1955 devant l'entrée principale (Marunouchi), en présence des familles de condamnés à mort. Cf. « Tōkyō no dōzō (16): Senpan no Ai no zō to Yokoe Yoshizumi », Tōkyō shinbun, 19 novembre 1959. 17. Plus généralement, un kigō (terme qu'on pourrait traduire littéralement par "geste au pinceau ») est une petite peinture ou calligraphie réalisée sur commande.

18. Ce qui est surtout vrai pour Yoshida qui a lui-même négocié et signé le Traité de San Francisco.

19. Cf. Katō Nobuyuki, op. cit., p. 160.

20. La stèle qui explique sur place l'origine et la destination du Mausolée précise que le lieu choisi correspond aussi au « centre du Japon » (Nihon no chūshinchi 日本の中心地).

21. Voir sur la construction du monument: Miura Shigeo 三浦重雄, Mikawa-wan kokutei kōen Sangane-san to junkoku nanashi no haka 三河湾国定公園三ヶ根山と殉国七士廟の墓, Gamagōri, Junkoku nanashi byō hōsankai, 1966.

22. Voir dans le présent numéro l'article de Takahashi Tetsuya, $\$ 3$.

23. Le Parc national de la baie de Mikawa fut institué en 1958, soit deux ans avant l'inauguration du Mausolée.

24. Le Front armé anti-japonais de l'Asie orientale (Higashi-Ajia hannichi busō sensen 東アジア 反日武装戦線) est un mouvement révolutionnaire qui fut actif de fin 1971 à 1974, date à laquelle il fusionna avec l'Armée rouge du Japon (Nihon sekigun). Ses principaux membres étaient Daidōji 
Masashi 大道時将司, Daidōji Ayako 大道寺あや子, Kataoka Toshiaki 片岡利明, Sasaki Norio 佐々 木規夫.

25. La dénomination même du Front armé ne fut décidée qu'en février 1972.

26. Les sept dirigeants en question morts en captivité sont Koiso Kuniaki, Hiranuma Kiichirô 平 沼騏一郎 (1867-1952), Tôgô Shigenori 東郷茂徳 (1882-1950), Matsuoka Yôsuke 松岡洋右 (1880-1946), Nagano Osami 永野修身 (1880-1947), Shiratori Toshio 白鳥敏夫 (1887-1949) et Umezu Yoshijirô 梅津美治郎 (1882-1949). Notons que Hiranuma est mort en août 1952, juste après la fin de l'occupation.

27. On pouvait lire en 2006 dans la revue de politique internationale de la Hoover Institution: «All told, more than 900 Japanese prisoners (as well as a few Koreans) were executed in Allied tribunals throughout the Far East. Many of their ashes are now interred in the Yasukuni Shrine in Tokyo "; Victor Martus, «On the disposal of dictators", Policy Review, n ${ }^{\circ} 134$, Hoover Institution, déc. 2005-jan. 2006.

28. Cf. Takahashi Tetsuya, Yasukuni mondai, op. cit., p. 73-74.

29. En français sur ces questions, voir, entre autres : Pierre Lavelle, «La Société pour la rédaction de nouveaux manuels d'histoire. Renouveau ou déclin du nationalisme ? ", Cipango, $\mathrm{n}^{\circ}$ 10, 2003 , p. 7-88; Arnaud Nanta, «Le débat sur l'enseignement de l'histoire au Japon ", Matériaux pour l'histoire de notre temps, $\mathrm{n}^{\circ} 88,2007, \mathrm{p} .13-19$; Eric Seizelet, "L'après-guerre comme paradigme politique », in M. Lucken, A. Bayard-Sakai, E. Lozerand (dir.), Le Japon après la guerre, Arles, Picquier, 2007, p. 19-46.

30. Cette œuvre est signée du sculpteur Katō Chōkō 加藤潮光 (1916-).

31. Tanaka Masaaki, « Kōa Kannon kaiki 60-nen », Kōa Kannon, n 12, 18 octobre 2000.

32. Pierre Lavelle, op. cit., p. 23.

33. Tokutomi Tasaburō, « Kōa Kannon wo kangaeru », Kōa Kannon, n 1, automne 1994.

34. Tanaka Masaaki, « Dare yori mo Chūgoku wo ai shita hito : Matsui Iwane », op. cit.

35. Michael Lucken, «Introduction », in M. Lucken, A. Bayard-Sakai, E. Lozerand (dir.), op. cit., p. 9.

36. Michael Lucken, «Le sort des statues et monuments commémoratifs durant l'occupation américaine ", op. cit., p. 141-158.

\section{RÉSUMÉS}

À la différence de la situation allemande, les restes des sept principaux criminels de guerre japonais ont été conservés et des monuments érigés à leur mémoire.

Unlike the situation in Germany, the remains of Japanese Class A War Criminals were kept and monuments were erected to their memory. 
INDEX

Mots-clés : mémoire, travail de mémoire, criminels de guerre

キーワード : sensō hanzainin 戦争犯罪人, kioku 記憶, Taiheiyō sensō 太平洋戦争 (1941-1945), sengo 戦後, shigakushi 史学史, rekishi 歴史

Index chronologique : guerre du Pacifique (1941-1945), après-guerre

Keywords : War Criminals, Memory, War of the Pacific (1939-1945), History, Historiography

Thèmes : historiographie, histoire 